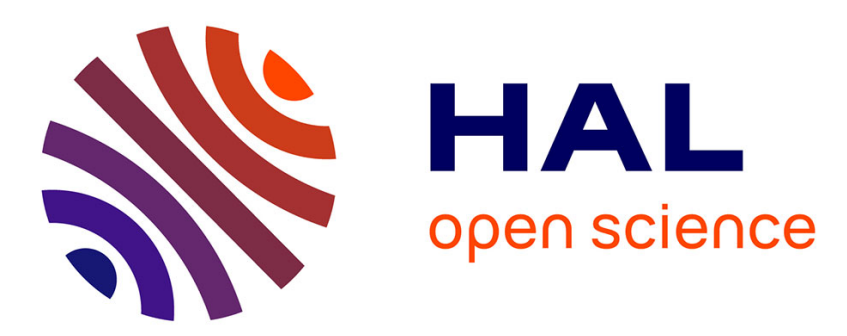

\title{
Rôle de la voie Sonic Hedgehog dans les cancers thoraciques
}

\author{
E. Giroux Leprieur, M. Antoine, T. Vieira, N. Rozensztajn, A.-M. Ruppert, \\ N. Rabbe, J. Cadranel, M. Wislez
}

\section{> To cite this version:}

E. Giroux Leprieur, M. Antoine, T. Vieira, N. Rozensztajn, A.-M. Ruppert, et al.. Rôle de la voie Sonic Hedgehog dans les cancers thoraciques. Revue des Maladies Respiratoires, 2015, 32 (8), pp.800-808. 10.1016/j.rmr.2014.11.069 . hal-01140278

\section{HAL Id: hal-01140278 https://hal.sorbonne-universite.fr/hal-01140278}

Submitted on 8 Apr 2015

HAL is a multi-disciplinary open access archive for the deposit and dissemination of scientific research documents, whether they are published or not. The documents may come from teaching and research institutions in France or abroad, or from public or private research centers.
L'archive ouverte pluridisciplinaire HAL, est destinée au dépôt et à la diffusion de documents scientifiques de niveau recherche, publiés ou non, émanant des établissements d'enseignement et de recherche français ou étrangers, des laboratoires publics ou privés. 
Rubrique : Revue générale

\section{Rôle de la voie Sonic Hedgehog dans les cancers thoraciques Role of the Sonic Hedgehog pathway in thoracic cancers}

Titre court : Rôle de la voie Sonic Hedgehog dans les cancers thoraciques

Etienne Giroux Leprieur ${ }^{1,2}$, Martine Antoine ${ }^{1}$, Thibault Vieira ${ }^{1,3}$, Nathalie Rozensztajn ${ }^{3}$, AnneMarie Ruppert ${ }^{1,3}$, Nathalie Rabbe ${ }^{1}$, Jacques Cadranel ${ }^{1,3}$, Marie Wislez $^{1,3}$

1. Sorbonne Universités, UPMC Université Paris 06, GRC n04, Theranoscan, F-75252, Paris, France

2. AP-HP, Hôpital Ambroise Paré, Service de Pneumologie et Oncologie Thoracique, Université Versailles Saint Quentin en Yvelines EA4340 BCOH, 92100 BoulogneBillancourt, France

3. AP-HP, Hôpital Tenon, Service de Pneumologie, F-75970, Paris, France

Soutiens : Fonds de Recherche en Santé Respiratoire (EGL)

Titre court : Rôle de la voie Shh dans les cancers thoraciques

Auteur correspondant: Marie Wislez, Hôpital Tenon, Service de Pneumologie, AP-HP, 75970, Paris, France. Tel: +33156016838. Fax: +33156017248. E-mail: marie.wislez@tnn.aphp.fr

\section{Conflit d'intérêt :}

Au cours des 5 dernières années, EGL a perçu des financements pour travaux de recherche de la part du Fond de Recherche en Santé Respiratoire. 


\section{Résumé}

Introduction : La voie de signalisation Sonic Hedgehog (Shh) est une voie physiologiquement activée durant l'embryogenèse et le développement. Elle jouerait un rôle dans la fibrose pulmonaire idiopathique, et est aussi réactivée dans plusieurs cancers solides. Etat des connaissances: La voie Shh est réactivée dans les cancers thoraciques, comme le cancer bronchique à petites cellules (CBPC), le cancer bronchique non à petites cellules (CBNPC) et le mésothéliome pleural malin (MPM). Cette voie est intimement liée aux cellules souches cancéreuses, et semble jouer un rôle important dans la prolifération tumorale, l'agressivité et la chimiorésistance de ces cancers. Cette revue décrit le mode d'activation de la voie Shh dans les cancers thoraciques, et son rôle dans les CBPC, les CBNPC et les MPM, à partir de modèles in vitro et in vivo. Les données de la littérature montrent que l'inhibition de la voie Shh a une action anti-tumorale et permet une sensibilisation à la chimiothérapie. Perspectives : Ces résultats incitent à développer des thérapies ciblant la voie Shh dans la prise en charge thérapeutique des cancers thoraciques.

Mots-clés : cancer bronchique à petites cellules, cancer bronchique non à petites cellules, mésothéliome, Sonic Hedgehog, chimiorésistance, vismodegib 


\begin{abstract}
Introduction: Sonic Hedgehog (Shh) pathway is physiologically activated during embryogenesis and development. It plays a role in idiopathic lung fibrosis, and is also activated in several solid cancers. State of the art: Shh pathway is reactivated in thoracic cancers, as small cell lung carcinoma (SCLC), non-small cell lung carcinoma (NSCLC) and malignant pleural mesothelioma (MPM). Shh pathway is associated with cancer stem cells, and seems to have a crucial role in tumor proliferation, aggressiveness and chemoresistance in these cancers. This review describes the activation mode of Shh pathway in thoracic cancers, and its role in SCLC, NSCLC and MPM, using in vitro and in vivo models. Notably, data from literature show that inhibition of Shh pathway has an antitumor action, and sensibilizes to chemotherapy. Perspectives: These results incite to develop targeted therapies against Shh pathway in the treatment of thoracic cancers
\end{abstract}

Key words: small cell lung carcinoma, non-small cell lung carcinoma, mesothelioma, Sonic Hedgehog, chemoresistance, vismodegib 


\section{- Introduction}

La voie de signalisation Sonic Hedgehog (Shh) est une voie physiologiquement activée durant l'embryogenèse et le développement [1]. Le gène hedgehog a été initialement découvert chez la drosophile en 1980 et décrit par les professeurs Wieschaus et Nüsslein-Volhard [2], ce qui leur a valu le prix Nobel en 1995. Le nom hedgehog (hérisson en anglais) est dû à l'aspect hérissé de la cuticule embryonnaire en cas de mutation de ce gène. Chez les mammifères, la voie Shh joue un rôle majeur dans le développement cérébral, des membres, et l'organogenèse pulmonaire. Elle est aussi réactivée dans un certain nombre de cancers solides, comme certains cancers cutanés [3-5], les médulloblastomes [6], les cancers pancréatiques, prostatiques, du sein [7-9], mais également dans les cancers thoraciques, que ce soit le cancer à petites cellules (CBPC) [10], le cancer bronchique non à petites cellules (CBNPC) [11-13] et le mésothéliome pleural malin (MPM) [14]. Son activation serait liée à la maintenance des cellules souches cancéreuses, et interviendrait probablement dès les premières étapes de la carcinogenèse $[15,16]$. De plus, elle serait associée à la résistance à la radiothérapie et à la chimiothérapie dans certains modèles de cancers. Le développement de traitements ciblés de cette voie pourrait être ainsi prometteur, notamment pour certains types de cancers réputés chimiorésistants.

Nous proposons, dans cette revue, de détailler les mécanismes d'activation de la voie Shh à l'état physiologique, dans la fibrose pulmonaire idiopathique, et ses mécanismes de réactivation en oncologie, en détaillant plus particulièrement les données de la littérature concernant les cancers thoraciques (CBPC, CBNPC et MPM).

\section{- La voie Shh}

\section{- $\quad$ La protéine Shh}

La voie de signalisation Hedgehog fait intervenir trois types de protéines : Indian, Desert et Sonic Hedghehog. Chez les mammifères, la protéine Sonic Hedgehog est celle qui intervient de manière préférentielle. Son gène, situé en 7q36, produit une pré-protéine de $45 \mathrm{kDa}$, qui va subir dans les conditions physiologiques un certain nombre de modifications post-transcriptionnelles [17]. Cette pré-protéine contient notamment en son sein un site d'auto-clivage, qui va permettre le clivage protéique en un résidu N-terminal (Shh-N) de $20 \mathrm{kDa}$ et un résidu C-terminal (Shh-C) de $25 \mathrm{kDa}$ [17]. La protéine Shh-C possède une activité cholestérol-transférase, qui va permettre l'adjonction de cholestérol à la partie C-terminale de Shh-N [18,19]. Secondairement, une nouvelle modification lipidique se produit avec ajout de résidus palmitoyl à la protéine Shh-N (palmitoylation). Toutes ces modifications lipidiques vont permettre à la protéine Shh de s'ancrer à la membrane cellulaire, avant d'être sécrétée dans l'espace extracellulaire [20]. En outre, la forme palmitoylée de Shh-N serait environ 30 fois plus active que sa forme non palmitoylée [21]. Toutes les fonctions physiologiques durant le développement sont liées à la forme Shh-N. La protéine Shh-C n'a pas de fonctions biologiques connues à l'état physiologique en dehors de son activité cholestérol-transférase, et est également sécrétée dans l'espace extracellulaire. 
- Activation de la voie Shh

Le récepteur membranaire de Shh est Patched (Ptch), dont il existe deux isoformes (Ptch1 et Ptch2). Ptch comprend 12 domaines transmembranaires. En l'absence de ligand (figure 1A), Ptch inhibe la migration à la membrane de la protéine Smoothened (Smo), en la maintenant à l'état inactif à l'intérieur de la cellule. Les facteurs de transcriptions Gli (Gli1, Gli2 et Gli3) sont alors liés à la protéine SUFU (Suppressor of fused homolog) et restent inactifs dans le cytoplasme [22]. Gli3 est également phosphorylé par la PKA (Protéine kinase A) et va ainsi produire une protéine inhibitrice GLIR qui a une action de répression de la transcription des gènes cibles de Shh.

Lorsque Shh se fixe à son récepteur Ptch (figure 1B), il y a levée de l'inhibition de Smo qui va alors migrer à la membrane. Les facteurs de transcription Gli se dissocient ensuite de SUFU pour migrer dans le noyau et activer la transcription des gènes cibles. On note qu'une fois que Shh est lié à Ptch, il y a une internalisation du complexe Shh-Ptch dans la cellule avant sa dégradation dans le lysosome [23].

- $\quad$ Phénomènes de rétrocontrôle négatif

À l'état physiologique, plusieurs phénomènes de rétrocontrôle négatif sont mis en jeu lors de l'activation de la voie Shh. Parmi ceux-ci, la protéine HHIP (Hedhegog Interacting Protein) est une protéine qui va se lier à Shh au niveau de la membrane cellulaire et ainsi entrer en compétition avec Ptch [24]. La liaison de Ptch avec son ligand Shh va également exercer un phénomène de contrôle négatif par phénomène d'endocytose [23]. Enfin, les protéines ZIC sont des facteurs de transcription, qui interagissent avec les protéines Gli via leur domaine à doigt de zinc [25], et ont une activité activatrice ou inhibitrice de Gli en fonction du type de cellule et des conditions [26].

- La protéine Shh se fixe à son récepteur membranaire Ptch, avec activation d'une autre protéine membranaire Smo, et activation des facteurs de transcription Gli.

- Des phénomènes de rétrocontrôle négatifs régulent à l'état physiologique la voie Shh.

\section{- Voie Shh dans le développement}

La voie Shh est capitale durant le développement embryonnaire [1]. Son activité est cependant dépendante du site de l'organisme où la voie est activée. Ainsi, chez le mammifère, Shh agit comme un morphogène au niveau du développement des membres, déterminant l'identité et le nombre des doigts. Il agit également comme morphogène pour le développement du système nerveux central au niveau de la crête neurale, avec un effet inducteur dans l'axe dorsoventral. Son action dans le développement pulmonaire est plus complexe. En effet, le développement de l'axe bronchique se fait par ramification. La mise en place de l'arbre aérien se fait entre la $4^{\mathrm{e}}$ et la $16^{\mathrm{e}}$ semaine, par ramification dichotomique à partir du bourgeon épithélial original. Plusieurs facteurs sont inducteurs de ce bourgeonnement, et sont produits par le mésenchyme à destination des cellules épithéliales. Il s'agit principalement du FGF-10 (Fibroblast Growth Factor-10), qui va se fixer à son récepteur FGFR2 (FGF Receptor-2) au niveau épithélial. Plusieurs facteurs interviennent comme régulateur de ce bourgeonnement épithélial. Certains sont externes à l'épithélium, comme le TGF $\beta$ (Transforming Growth Factor $\beta$ ), ou ont une action interne à 
l'épithélium, comme Sprouty2 ou Wnt (Wingless Integration site), mais les principaux sont sécrétés par l'épithélium à destination du mésenchyme, comme Shh ou BMP4 (Bone Morphogenic Protein 4). Ainsi, Shh réprime fortement l'expression de FGF-10 dans le mésenchyme (Figure 2). L'apparition de nouveaux foyers sécréteurs de FGF-10 provoque le bourgeonnement dichotomique. Shh agit donc comme régulateur spatial de FGF-10 durant le développement de l'arbre bronchique. L'utilisation de modèles murins knockout pour différents gènes de la voie Shh (modèles $\mathrm{Shh}^{-/}, \mathrm{Ptchl}^{-/}, \mathrm{Gli}^{-/-}$et $\mathrm{Gli3}^{-/}$) induit des défauts sévères de développement pulmonaire, avec hypoplasie pulmonaire et malformation trachéale, avec des phénotypes non viables à la naissance [27-32].

- La voie Shh joue un rôle majeur pendant l'embryogenèse et le développement.

- Lors du développement pulmonaire et la bronchogenèse, la protéine Shh est sécrétée par l'épithélium et agit au niveau du mésenchyme.

- Shh joue un rôle de régulateur du bourgeonnement épithélial en réprimant l'expression de FGF-10 dans le mésenchyme.

\section{- Voie Shh et fibrose pulmonaire idiopathique}

La fibrose pulmonaire idiopathique (FPI) est caractérisée sur le plan physiopathologique par des lésions épithéliales qui vont entraîner des phénomènes de transition épithélio-mésenchymateuse [33], avec activation et prolifération des cellules fibroblastiques, induisant des dépôts de collagène avec destruction de l'architecture alvéolaire pulmonaire [34]. Plusieurs études ont montré une activation aberrante de la voie Shh au cours de la FPI. Ainsi, des études d'expression génique par microarray sur des tissus pulmonaires de FPI ont mis en évidence une surexpression de Ptch1 [35]. Une surexpression de Shh, Smo et Gli1 a également été montrée, que ce soit par immunohistochimie (IHC), PCR quantitative, ou hybridation in situ, dans les poumons atteints de FPI [35-40]. De manière intéressante, l'expression de Shh semble être limitée aux cellules épithéliales, tandis que les facteurs d'aval de la voie Shh (Ptch1 et Gli1) sont exprimés à la fois dans les cellules épithéliales et les cellules mésenchymateuses, suggérant un signal agissant à la fois sur l'épithélium respiratoire lésé et le mésenchyme.

Plusieurs modèles animaux de fibrose pulmonaire ont confirmé le rôle de la voie Shh dans cette pathologie. Ainsi, sur des modèles de fibrose induite par la bléomycine, il existe une induction de l'expression de Shh et Gli1 [41], et l'induction de l'expression de Shh aggrave les lésions fibrosantes [42]. À l'inverse, l'inhibition de Gli par GANT61 inhibe la progression de la fibrose [41]. L'utilisation d'un rapporteur de l'expression de Gli1 (souris Gli1 ${ }^{\text {nlacZ/+ }}$ ) a permis de montrer que les cellules activées par Shh étaient majoritairement des fibroblastes et des myofibroblastes, et que ces cellules exprimant Gli1 étaient beaucoup plus nombreuses après instillation de bléomycine en comparaison avec les poumons non lésés [42]. Des résultats similaires ont été mis en évidence dans d'autres modèles de fibrose, comme le modèle de fibrose induite par le FITC [37].

- La voie Shh joue un rôle dans la physiopathologie de la FPI.

- Shh est sécrétée par l'épithélium lésé et va activer les fibroblastes et les myofibroblastes. 
- L'inhibition de la voie Shh a une action anti-fibrosante dans des modèles de fibrose induite par la bléomycine.

\section{- Voie Shh et cancer}

- Généralités

- $\quad$ Principaux mécanismes d'activation de Shh dans les cancers solides

La voie Shh est réactivée dans un certain nombre de cancers solides. Plusieurs mécanismes ont été décrits. La voie Shh peut ainsi être activée via la présence de mutations somatiques au niveau des différents effecteurs de cette voie. Ainsi, le syndrome de Gorlin, qui induit la formation de carcinomes basocellulaires multiples, de rhabdomyosarcomes et de médulloblastomes, est caractérisé par la présence de mutation constitutionnelle inactivatrice de Ptch, qui induit donc une activation continue de Smo [3,4]. Des mutations de $S U F U$ ont également été décrites dans les médulloblastomes sporadiques [6]. Dans les formes sporadiques de carcinomes basocellulaires, il existe une mutation de la voie Shh (mutation inactivatrice de Ptch ou mutation activatrice de Smo) dans environ $70 \%$ des cas [5]. L'importance de l'activation de la voie Shh dans ce type de cancer a permis le développement et la mise sur le marché d'un inhibiteur de Smo (vismodegib, Erivedge ${ }^{\circledR}$ (Genentech, USA)) dans le traitement des carcinomes basocellulaires [43].

Dans de nombreux autres cancers, une activation de la voie Shh a été décrite sans mise en évidence de mutation de la voie Shh. Il s'agit alors d'un mécanisme d'activation par voie auto- et paracrine, via la sécrétion de la protéine Shh. Un tel mécanisme d'activation a été décrit dans les cancers du sein, du pancréas et de la prostate [7-9].

- Gènes cibles de l'activation de Shh dans le cancer

Les gènes activés par les facteurs de transcription Gli sont très différents en fonction du contexte et du type de cellule concerné [44]. La plupart des gènes sont impliqués dans la prolifération, la survie cellulaire, la transition épithélio-mésenchymateuse (TEM) et le phénotype de cellule souche cancéreuse (CSC). Il existe également des gènes cibles de Gli qui vont exercer un rétrocontrôle négatif de la voie Shh. Le tableau 1 résume les principaux gènes cibles de l'activation de la voie Shh.

- $\quad$ Voie Shh et CSC

Plusieurs travaux ont montré une relation étroite entre la voie Shh et les CSC. En effet, Shh est une des voies de signalisation préférentiellement activée au sein des CSC, tout comme les voies Wnt et Notch. Plusieurs marqueurs de CSC ont été développés pour caractériser et isoler ces cellules, comme des marqueurs membranaires (CD133, CD44, BCRP) [45]. La capacité d'efflux du Hoechst 33342 ("side population " (SP)), c'est-à-dire le transport actif du Hoechst 33342 du milieu intracellulaire vers le milieu extracellulaire, via notamment des transporteurs ABC, a également été utilisée comme marqueur de CSC [46]. Plus récemment, de nombreux travaux ont 
validé l'activité ALDH (aldéhyde déshydrogénase) comme marqueur de CSC, notamment dans le CBNPC $[47,48]$. ALDH est un groupe d'enzymes responsables de l'oxydation des aldéhydes. Leur activité semble augmentée dans les cellules souches. Ainsi, leur suractivité est impliquée dans le métabolisme alcoolique, le métabolisme de la vitamine $\mathrm{A}$, la résistance à certains agents de chimiothérapie (cyclophosphamide...) et la différenciation précoce des cellules souches [49].

La relation entre les CSC et la voie Shh a été bien documentée dans la littérature. Ainsi, Li et coll. [14] ont isolé au sein de cultures primaires d'adénocarcinomes pancréatiques des cellules ayant un phénotype de CSC $(\mathrm{CD} 44(+) \mathrm{CD} 24(+) \mathrm{ESA}(+))$. Ces cellules avaient un potentiel d'oncogenèse 100 fois supérieur aux autres cellules, mesuré par la formation de xénogreffes sur souris immunodéprimés. Ces cellules avaient une surexpression de la voie Shh par rapport aux autres cellules tumorales. Dans un autre travail portant sur des lignées cellulaires de cancer bronchique (HCC et H1339), l'inhibition de la voie Shh par vismodegib entraînait une diminution de la population de CSC [50] mesurée la capacité d'efflux du Hoechst 33342 (cellules SP+) par analyse en cytométrie de flux. Le taux de cellules SP+ passait de 0,45\% (HCC) et 0,75\% (H13339) à 0,24\% (HCC) et $0,18 \%$ (H1339) après traitement par le vismodegib [50]. Des résultats similaires ont été mis en évidence dans des modèles de glioblastomes PTEN-dépendants [51]. Enfin, Lemjabbar-Alaoui et coll. [16] ont étudié un modèle de carcinogenèse bronchique induite par la fumée de cigarette dans lequel des cellules bronchiques humaines issues de cultures primaires, étaient exposées pendant 8 jours à la fumée de tabac. À l'issue de cette exposition, les cellules bronchiques avaient acquis un phénotype tumoral, avec augmentation de leur prolifération, de leur capacité de croissance en agarose, et de leur capacité de former des xénogreffes tumorales sur souris immunodéprimées. Il existait une activation des voies de signalisation Wnt et Shh dans ces cellules. L'inhibition de la voie Shh prévenait l'apparition du phénotype tumoral des cellules exposées à la fumée. Ces résultats soulignent le rôle majeur probable de la voie Shh dans l'oncogenèse des CBNPC.

- $\quad$ Activation de la voie Shh dans le CBPC

Watkins et coll. [10] ont pu montrer que la voie Shh était activée dans les CBPC, avec un mécanisme d'activation auto- et juxtacrine. En effet, l'étude de l'expression de Shh et Gli1 par immunohistochimie et Western Blot sur des prélèvements humains et des lignées cellulaires de CBPC a mis en évidence une surexpression de ces protéines par les cellules tumorales. L'inhibition de la voie Shh par la cyclopamine (inhibiteur de Smo, Figure 1B) inhibait la croissance tumorale des cellules de CBPC in vitro et in vivo sur des xénogreffes. Ces résultats ont été confirmés sur des modèles murins transgéniques, porteuses d'une délétion de Rbl(retinoblastoma 1) et de TRP53 (transformation related protein 53) [52]. La délétion de Smo dans ces modèles, ainsi que l'utilisation d'inhibiteurs pharmacologiques de la voie Shh, inhibaient la croissance tumorale.

- $\quad$ Activation de la voie Shh dans le CBNPC 
La voie Shh est également réactivée dans le CBNPC. Yuan et coll. [11] ont montré une activation de la voie Shh dans un certain nombre de lignées cellulaires de CBNPC. Ils ont également analysés l'expression de Gli1 par immunohistochimie sur un panel de 120 échantillons de cancers bronchiques (tissue microarray). Ils ont ainsi mis en évidence une positivité dans $87 \%$ des adénocarcinomes et $93 \%$ des carcinomes épidermoïdes. Une autre étude par analyse immunohistochimique des différents composés de la voie Shh sur 80 pièces chirurgicales de CBNPC (stade I à III) a également montré une expression de Shh (97,5 \%), Ptch1 (78,7 \%), Smo (72,5\%), Gli1 (98,7\%) et Gli2 (87,5\%) [12]. L'expression était présente uniquement dans les cellules tumorales. Les tissus normaux péritumoraux étaient négatifs. Dans une autre étude de 248 CBNPC de stade précoce, l'expression des différents composés de la voie Shh n'était pas corrélée à la survie sans récidive ou à la survie globale [13].

L'hypothèse d'un rôle protumoral de la voie Shh dans les CBNPC a été confirmée dans une étude récente [53]. Les auteurs ont analysé l'expression de la voie Shh, par PCR sur deux cohortes différentes de patients ayant un carcinome épidermoïde bronchique $(n=178$ et $n=56)$, ainsi que sur quatre lignées de carcinome épidermoïde bronchique (H520, H2170, H226, et SK-MES-1). La voie Shh était activée dans les prélèvements de ces deux cohortes. In vitro, l'inhibition de la voie Shh, notamment l'inhibition de Gli2 par siRNA ou par une molécule inhibitrice (GANT61), diminuait la prolifération et activait l'apoptose. L'utilisation de GANT61 in vivo sur des modèles de xénogreffes montrait également une activité antitumorale.

Concernant le rôle de la voie Shh dans la chimiorésistance, il a été montré une corrélation entre l'activation de la voie Shh dans les cellules cancéreuses et l'expression de transporteurs ABC (ATP-binding cassette) impliqués dans la chimiorésistance, comme MDR1 et ABCG2 [54]. L'acquisition d'un phénotype mésenchymateux par les cellules tumorales (transition épithéliomésenchymateuse), connu comme facteur de chimiorésistance, a également été montrée comme étant associée à l'activation de la voie Shh. Ainsi, il existe une corrélation inverse entre l'expression de Gli et celle des marqueurs épithéliaux comme l'E-cadhérine [55]. Plusieurs études ont testé l'association Shh et chimiorésistance in vitro et in vivo. Tian et coll. [56] ont montré que, sur des lignées cellulaires de cancer bronchique résistantes au cisplatine, le vismodegib inhibait de façon significative la prolifération tumorale in vitro. L'inhibition de la voie Shh par vismodegib ou par siRNA sensibilise également aux inhibiteurs de tyrosine kinase (ITK) de 1'EGFR. Ahmad et coll. [57] ont utilisé la lignée A549, rendue résistante au cisplatine et à l'erlotinib via un traitement par TGF $\beta$ qui permet l'acquisition d'un phénotype mésenchymateux (A549M). Les IC50 du cisplatine et de l'erlotinib étaient significativement plus basses avec un prétraitement par vismodegib ou siRNA anti-Shh. L'administration de vismodegib entraînait la diminution de l'expression de marqueurs de CSC, comme Sox2 ou Nanog.

\section{- $\quad$ Activation de la voie Shh dans le MPM}

De manière analogue, une surexpression des protéines de la voie Shh a été mise en évidence dans le MPM, tandis que le tissu pleural normal ne les exprime pas [13]. Par ailleurs, sur des modèles de cultures primaires de MPM, l'inhibition de Smo entraîne une diminution significative de la croissance tumorale via l'inhibition de Glil. Enfin, l'inhibition de Smo a également une action 
antitumorale in vivo sur des modèles de xénogreffes animales. Des résultats similaires ont été obtenus par l'inhibition directe de Gli par siRNA ou l'utilisation d'une petite molécule inhibitrice de Gli (Gli-I) [58] ou de GANT61 [59]. Par ailleurs, de manière intéressante, l'inhibition de Gli permet une potentialisation de l'action cytotoxique de la chimiothérapie in vitro. La molécule Gli-I a un effet cytotoxique synergique avec le pemetrexed sur des lignées de MPM[58].

- La voie Shh est réactivée dans les cancers thoraciques, que ce soit le CBPC, le CBNPC et le mésothéliome pleural.

- La voie Shh semble liée aux cellules souches cancéreuses.

- Elle jouerait un rôle dans la prolifération tumorale, mais aussi dans la chimiorésistance et la TEM.

- L'inhibition de la voie Shh a une activité antitumorale in vitro et in vivo.

\section{- Perspectives thérapeutiques}

Actuellement, les inhibiteurs de la voie Sonic Hedgehog développés en clinique sont tous des inhibiteurs de Smo (Tableau 2). Le vismodegib a actuellement 1'AMM dans le traitement des carcinomes basocellulaires. En oncologie thoracique, le vismodegib a été testé par l'Eastern Cooperative Oncology Group en phase II dans le CBPC (ECOG-1508) qui évaluait l'intérêt de l'ajout du vismodegib à une chimiothérapie par cisplatine et étoposide dans les CBPC disséminés, en première ligne de traitement. Aucune différence en terme de survie sans progression, ni de survie globale n'a été mise en évidence entre les patients ayant reçu une chimiothérapie seule et ceux traités par vismodegib en plus [60]. Plusieurs hypothèses peuvent expliquer ces résultats négatifs : l'absence de sélection d'un phénotype de patients particuliers (l'inhibition de la voie Shh n'est-elle bénéfique que pour les patients chimiorésistants ?) l'absence de biomarqueur permettant d'identifier les patients susceptibles de bénéficier du vismodegib ou encore le modèle de traitement concomitant (chimiothérapie et vismodegib) utilisé pour cet essai. Les autres inhibiteurs de Smo sont actuellement en cours d'évaluation en phase I dans les cancers solides.

\section{- Conclusion}

Outre son rôle bien connu lors de l'embryogenèse, la voie Shh joue également un rôle protumoral dans un certain nombre de cancers solides, comme les cancers thoraciques. Elle est réactivée dans les CBPC, les CBNPC et les MPM, par un mécanisme d'activation auto- et juxtacrine, sans qu'actuellement aucune des mutations somatiques connues n'aient été mise en évidence. La voie Shh, intimement liée aux CSCs et à la carcinogenèse, est également corrélée à la résistance aux traitements anticancéreux, comme la chimiothérapie cytotoxique, et possiblement aux thérapies ciblées comme les ITK de l'EGFR. L'inhibition de la voie Shh représente donc une approche prometteuse dans la prise en charge des cancers thoraciques, et des essais thérapeutiques sont nécessaires dans le CBNPC et le MPM. 


\section{Points forts}

- La voie Shh joue un rôle majeur lors de l'embryogenèse, notamment durant le développement pulmonaire.

- La voie Shh intervient dans la FPI, et son inhibition a une action antifibrosante.

- La voie Shh est réactivée dans les cancers thoraciques (CBPC, CBNPC, mésothéliome pleurale). Elle joue un rôle dans la prolifération tumorale, est associée à la TEM et à la chimiorésistance.

- L'inhibition de la voie Shh permet une sensibilisation à la chimiothérapie in vitro.

- L'inhibition de la voie Shh a une action antitumorale in vitro et in vivo.

- La recherche clinique sur l'effet de l'inhibition de la voie Shh dans la prise en charge des cancers thoraciques doit être encouragée.

\section{Références}

1. McMahon AP, Ingham PW, Tabin CJ. Developmental roles and clinical significance of hedgehog signaling. Curr Top Dev Biol 2003 ; 53 : 1-114.

2. Nüsslein-Volhard C, Wieschaus E. Mutations affecting segment number and polarity in Drosophila. Nature $1980 ; 287: 795-801$.

3. Hahn H, Wicking C, Zaphiropoulous PG, Gailani MR, Shanley S, Chidambaram A, et al. Mutations of the human homolog of Drosophila patched in the nevoid basal cell carcinoma syndrome. Cell 1996; 85 : 841 51.

4. Johnson RL, Rothman AL, Xie J, Goodrich LV, Bare JW, Bonifas JM, et al. Human homolog of patched, a candidate gene for the basal cell nevus syndrome. Science $1996 ; 272$ : 1668-71.

5. Tang JY, So PL, Epstein EH Jr. Novel Hedgehog pathway targets against basal cell carcinoma. Toxicol Appl Pharmacol 2007 ; 224 : 257-64.

6. Raffel C, Jenkins RB, Frederick L, Hebrink D, Alderete B, Fults DW, et al. Sporadic medulloblastomas contain PTCH mutations. Cancer Res 1997 ; 57 : 842-5.

7. Karhadkar SS, Bova GS, Abdallah N, Dhara S, Gardner D, Maitra A, et al. Hedgehog signalling in prostate regeneration, neoplasia and metastasis. Nature 2004 ; 431 : 707-12.

8. Kubo M, Nakamura M, Tasaki A, Yamanaka N, Nakashima H, Nomura M, et al. Hedgehog signaling pathway is a new therapeutic target for patients with breast cancer. Cancer Res 2004 ; 64 : 6071-4.

9. Thayer SP, di Magliano MP, Heiser PW, Nielsen CM, Roberts DJ, Lauwers GY, et al. Hedgehog is an early and late mediator of pancreatic cancer tumorigenesis. Nature 2003 ; 425 : 851-6.

10. Watkins DN, Berman DM, Burkholder SG, Wang B, Beachy PA, Baylin SB. Hedgehog signalling within 
airway epithelial progenitors and in small-cell lung cancer. Nature 2003 ; 422 : 313-7.

11. Yuan Z, Goetz JA, Singh S, Ogden SK, Petty WJ, Black CC, et al. Frequent requirement of hedgehog signaling in non-small cell lung carcinoma. Oncogene 2007 ; 26 : 1046-55.

12. Gialmanidis IP, Bravou V, Amanetopoulou SG, Varakis J, Kourea H, Papadaki H. Overexpression of hedgehog pathway molecules and FOXM1 in non-small cell lung carcinomas. Lung Cancer 2009 ; 66 : 64-74.

13. Raz G, Allen KE, Kingsley C, Cherni I, Arora S, Watanabe A, et al. Hedgehog signaling pathway molecules and ALDH1A1 expression in early-stage non-small cell lung cancer. Lung Cancer 2012 ; 76 : 191-6.

14. Shi Y, Moura U, Opitz I, Soltermann A, Rehrauer H, Thies S, et al. Role of hedgehog signaling in malignant pleural mesothelioma. Clin Cancer Res 2012 ; 18 : 4646-56.

15. Li C, Heidt DG, Dalerba P, Burant CF, Zhang L, Adsay V, et al. Identification of pancreatic cancer stem cells. Cancer Res 2007 ; 67 : 1030-7.

16. Lemjabbar-Alaoui H, Dasari V, Sidhu SS, Mengistab A, Finkbeiner W, Gallup M, et al. Wnt and Hedgehog are critical mediators of cigarette smoke-induced lung cancer. PLoS One 2006; 1 : e93.

17. Lee JJ, Ekker SC, von Kessler DP, Porter JA, Sun BI, Beachy PA. Autoproteolysis in hedgehog protein biogenesis. Science 1994 ; 266 : 1528-37.

18. Pepinsky RB, Zeng C, Wen D, Rayhom P, Baker DP, Williams KP, et al. Identification of a palmitic acidmodified form of human Sonic hedgehog. J BiolChem 1998 ; 273 : 14037-45.

19. Porter JA, Young KE, Beachy PA. Cholesterol modification of hedgehog signaling proteins in animal development. Science $1996 ; 274$ : 255-9.

20. Robbins DJ, Hebrok M. Hedgehogs: la dolce vita. Workshop on Hedgehog-Gli Signaling in Cancer and Stem Cells. EMBO Rep 2007 ; 8 : 451-5.

21. Wendler F, Franch-Marro X, Vincent JP. How does cholesterol affect the way Hedgehog works? Development $2006 ; 133$ : 3055-61.

22. Kogerman P, Grimm T, Kogerman L, Krause D, Undén AB, Sandstedt B, et al. Mammalian suppressorof-fused modulates nuclear-cytoplasmic shuttling of Gli-1. Nat Cell Biol 1999; 1 : 312-9.

23. Torroja C, Gorfinkiel N, Guerrero I. Patched controls the Hedgehog gradient by endocytosis in a dynamindependent manner, but this internalization does not play a major role in signal transduction. Development $2004 ; 131: 2395-408$.

24. Chuang PT, McMahon AP. Vertebrate Hedgehog signalling modulated by induction of a Hedgehogbinding protein. Nature $1999 ; 397: 617-21$.

25. Koyabu Y, Nakata K, Mizugishi K, Aruga J, Mikoshiba K. Physical and functional interactions between Zic and Gli proteins. J Biol Chem 2001 ; 276 : 6889-92.

26. Mizugishi K, Aruga J, Nakata K, Mikoshiba K. Molecular properties of Zic proteins as transcriptional 
regulators and their relationship to GLI proteins. J Biol Chem 2001 ; 276 : 2180-8.

27. Bai CB, Auerbach W, Lee JS, Stephen D, Joyner AL. Gli2, but not gli1, is required for initial shh signaling and ectopic activation of the shh pathway. Development $2002 ; 129: 4753-4761$.

28. Motoyama J, Liu J, Mo R, Ding Q, Post M, Hui CC. Essential function of gli2 and gli3 in the formation of lung, trachea and oesophagus. Nat Genet 1998 ; $20: 54-57$.

29. Grindley JC, Bellusci S, Perkins D, Hogan BL. Evidence for the involvement of the gli gene family in embryonic mouse lung development. Dev Biol 1997 ; 188 : 337-348.

30. Pepicelli CV, Lewis PM, McMahon AP. Sonic hedgehog regulates branching morphogenesis in the mammalian lung. Curr Biol 1998 ; 8 : 1083-1086.

31. Litingtung Y, Lei L, Westphal H, Chiang C. Sonic hedgehog is essential to foregut development. Nat Genet $1998 ; 20: 58-61$.

32. Goodrich LV, Milenkovic L, Higgins KM, Scott MP. Altered neural cell fates and medulloblastoma in mouse patched mutants. Science 1997 ; 277 : 1109-1113.

33. Kim KK, Kugler MC, Wolters PJ, Robillard L, Galvez MG, Brumwell AN, et al. Alveolar epithelial cell mesenchymal transition develops in vivo during pulmonary fibrosis and is regulated by the extracellular matrix. Proc Natl Acad Sci U S A 2006 ; 103 : 13180-5.

34. Fernandez IE, Eickelberg O. New cellular and molecular mechanisms of lung injury and fibrosis in idiopathic pulmonary fibrosis. Lancet $2012 ; 380: 680-688$.

35. Selman M, Pardo A, Kaminski N. Idiopathic pulmonary fibrosis: Aberrant recapitulation of developmental programs? PLoS Med 2008 ; 5 : e62.

36. Coon DR, Roberts DJ, Loscertales M, Kradin R. Differential epithelial expression of shh and foxf1 in usual and nonspecific interstitial pneumonia. Exp Mol Pathol 2006 ; 80 : 119-123.

37. Cigna N, Moshai EF, Brayer S, Marchal-Somme J, Wemeau-Stervinou L, Fabre A, et al. The hedgehog system machinery controls transforming growth factor-beta-dependent myofibroblastic differentiation in humans: Involvement in idiopathic pulmonary fibrosis. Am J Pathol 2012 ; 181 : 2126-2137.

38. Stewart GA, Hoyne GF, Ahmad SA, Jarman E, Wallace WA, Harrison DJ, et al. Expression of the developmental sonic hedgehog (shh) signalling pathway is up-regulated in chronic lung fibrosis and the shh receptor patched 1 is present in circulating t lymphocytes. J Pathol 2003 ; 199 : 488-495.

39. Fitch PM, Howie SE, Wallace WA. Oxidative damage and TGF-beta differentially induce lung epithelial cell sonic hedgehog and tenascin-c expression: Implications for the regulation of lung remodelling in idiopathic interstitial lung disease. Int J Exp Pathol 2011 ; 92 : 8-17.

40. Bolanos AL, Milla CM, Lira JC, Ramirez R, Checa M, Barrera L, et al. Role of sonic hedgehog in idiopathic pulmonary fibrosis. Am J Physiol Lung Cell Mol Physiol 2012, 303 : L978-90..

41. Moshai EF, Wemeau-Stervinou L, Cigna N, Brayer S, Somme JM, Crestani B, et al. Targeting the hedgehog-gli pathway inhibits bleomycin induced lung fibrosis in mice. Am J Respir Cell Mol Biol 2014 ; 51 : 11-25.. 
42. Liu L, Kugler MC, Loomis CA, Samdani R, Zhao Z, Chen GJ, et al. Hedgehog signaling in neonatal and adult lung. Am J Respir Cell Mol Biol 2013 ; 48 : 703-710.

43. Sekulic A, Migden MR, Oro AE, Dirix L, Lewis KD, Hainsworth JD, et al. Efficacy and safety of vismodegib in advanced basal-cell carcinoma. N Engl J Med $2012 ; 366$ : 2171-9.

44. Katoh Y, Katoh M. Hedgehog target genes: mechanisms of carcinogenesis induced by aberrant hedgehog signaling activation. Curr Mol Med 2009 ; 9: 873-86.

45. Rivera C, Rivera S, Loriot Y, Vozenin MC, Deutsch E. Lung cancer stem cell: new insights on experimental models and preclinical data. J Oncol 2011 ; 2011 : 549181.

46. Ho MM, Ng AV, Lam S, Hung JY. Side population in human lung cancer cell lines and tumors is enriched with stem-like cancer cells. Cancer Res 2007 ; 67 : 4827-33.

47. Jiang F, Qiu Q, Khanna A, Todd NW, Deepak J, Xing L, et al. Aldehyde dehydrogenase 1 is a tumor stem cell-associated marker in lung cancer. Mol Cancer Res $2009 ; 7$ :330-8.

48. Liang D, Shi Y. Aldehyde dehydrogenase-1 is a specific marker for stem cells in human lung adenocarcinoma. Med Oncol $2012 ; 29: 633-9$.

49. Storms RW, Trujillo AP, Springer JB, Shah L, Colvin OM, Ludeman SM, et al. Isolation of primitive human hematopoietic progenitors on the basis of aldehyde dehydrogenase activity. Proc Natl Acad Sci US A 1999; 96:9118-23.

50. Tian F, Mysliwietz J, Ellwart J, Gamarra F, Huber RM, Bergner A. Effects of the Hedgehog pathway inhibitor GDC-0449 on lung cancer cell lines are mediated by side populations. Clin Exp Med 2012; 12 : 25-30.

51. Xu Q, Yuan X, Liu G, Black KL, Yu JS. Hedgehog signaling regulates brain tumor-initiating cell proliferation and portends shorter survival for patients with PTEN-coexpressing glioblastomas. Stem Cells $2008 ; 26: 3018-26$.

52. Park KS, Martelotto LG, Peifer M, Sos ML, Karnezis AN, Mahjoub MR, et al. A crucial requirement for Hedgehog signaling in small cell lung cancer. Nat Med $2011 ; 17: 1504-8$.

53. Huang L, Walter V, Hayes DN, Onaitis M. Hedgehog-GLI Signaling Inhibition Suppresses Tumor Growth in Squamous Lung Cancer. Clin Cancer Res 2014 ; 20 : 1566-75.

54. Sims-Mourtada J, Izzo JG, Ajani J, Chao KS. Sonic Hedgehog promotes multiple drug resistance by regulation of drug transport. Oncogene 2007 ; 26 : 5674-9.

55. Yue D, Li H, Che J, Zhang Y, Tseng HHK, Jin JQ, et al. Hedgehog/Gli promotes epithelial-mesenchymal transition in lung squamous cell carcinomas. J Exp Clin Cancer Res 2014 ; 33:34.

56. Tian F, Schrödl K, Kiefl R, Huber RM, Bergner A. The hedgehog pathway inhibitor GDC-0449 alters intracellular $\mathrm{Ca} 2+$ homeostasis and inhibits cell growth in cisplatin-resistant lung cancer cells. Anticancer Res $2012 ; 32$ : 89-94.

57. Ahmad A, Maitah MY, Ginnebaugh KR, Li Y, Bao B, Gadgeel SM, et al. Inhibition of Hedgehog signaling sensitizes NSCLC cells to standard therapies through modulation of EMT-regulating miRNAs. $J$ 
Hematol Oncol $2013 ; 6: 77$.

58. Li H, Lui N, Cheng T, Tseng HH, Yue D, Giroux Leprieur E, et al. Gli as a novel therapeutic target in malignant pleural mesothelioma. PLoS One 2013 ; 8 : e57346.

59. You M, Varona-Santos J, Singh S, Robbins DJ, Savaraj N, Nguyen DM. Targeting of the Hedgehog signal transduction pathway suppresses survival of malignant pleural mesothelioma cells in vitro. J Thorac Cardiovasc Surg 2014 ; 147 : 508-16.

60. Belani CK, Dahlberg SE, Rudin CM, Fleisher M, Chen HX, Takebe N, et al. Three-arm randomized phase II study of cisplatin and etoposide (CE) versus $\mathrm{CE}$ with either vismodegib (V) or cixutumumab (Cx) for patients with extensive stage-small cell lung cancer (ES-SCLC) (ECOG 1508). J Clin Oncol 2013 ; 31 suppl : abstr 7508.

61. Affolter M, Zeller R, Caussinus E. Tissue remodelling through branching morphogenesis. Nat Rev Mol Cell Biol 2009; 10 : 831-42. 
Tableau 1 : Principaux gènes cibles de la voie Shh. D'après Katoh [44]

\begin{tabular}{|c|c|}
\hline & Gène \\
\hline \multirow[t]{5}{*}{ TEM } & FOXC2 \\
\hline & SNAII \\
\hline & TWIST2 \\
\hline & ZEB1 \\
\hline & ZEB2 \\
\hline \multirow[t]{11}{*}{ CSC } & $J A G 2$ \\
\hline & $F S T$ \\
\hline & GREM1 \\
\hline & BMP4 \\
\hline & $W N T 2 B$ \\
\hline & WNT5A \\
\hline & PDGFRA \\
\hline & BMII \\
\hline & $L G R 5$ \\
\hline & $C D 44$ \\
\hline & $C D 133$ \\
\hline rétrocontrôle positif de la voie Shh & GLII \\
\hline \multirow[t]{3}{*}{ rétrocontrôle négatif de la voie Shh } & PTCH1 \\
\hline & PTCH2 \\
\hline & HHIP1 \\
\hline \multirow[t]{7}{*}{ prolifération cellulaire } & $M Y C N$ \\
\hline & CCND1 \\
\hline & $C C N D 2$ \\
\hline & CCNE \\
\hline & FOXM1 \\
\hline & $C C N B 1$ \\
\hline & $C D C 25 B$ \\
\hline \multirow[t]{2}{*}{ survie cellulaire } & $B C L 2$ \\
\hline & CFLAR \\
\hline \multirow[t]{4}{*}{ autres } & $F O X F 1$ \\
\hline & FOXL1 \\
\hline & PRDM1 \\
\hline & PTHLH \\
\hline
\end{tabular}


Tableau 2 : Principaux inhibiteurs de Smo en développement

\begin{tabular}{|l|l|}
\hline \multicolumn{1}{|c|}{ Produit } & \multicolumn{1}{|c|}{ Laboratoire pharmaceutique } \\
\hline vismodegib (GDC-0449) & Genentech \\
\hline BMS-833923 & Bristol-Meyers-Squibb \\
\hline IPI-926 & Infinity Pharmaceuticals \\
\hline LDE225 & Novartis \\
\hline LEQ506 & Novartis \\
\hline PF-04449913 & Pfizer \\
\hline TAK-441 & Millennium Pharmaceuticals \\
\hline
\end{tabular}


Légendes des figures

Figure 1 : Voie de signalisation Shh. A : en l'absence de fixation de Shh sur Ptch, Ptch exerce une action inhibitrice sur Smo. Gli est associé à SUFU dans le cytoplasme et est inactif. B : en cas de fixation de Shh sur Ptch, il y a levée de l'inhibition de Smo et activation de Gli qui va passer dans le noyau et activer la transcription des gènes cibles. Plusieurs inhibiteurs de la vois Shh existent : inhibiteurs de Smo (vismodegib, cyclopamine) et inhibiteurs de Gli (GANT61, petite molécule inhibitrice de Gli (Gli-I)).

Figure 2 : Interactions épithélium - mésenchyme lors du bourgeonnement et de la ramification bronchique au cours du développement, et rôle de Shh. La croissance épithéliale est stimulée par la sécrétion de FGF-10 par le mésenchyme. Des facteurs régulateurs vont intervenir, comme Shh, Sprouty (Spry2), TGF $\beta$, Wnt et BMP4. Shh va ainsi être sécrétée par l'épithélium dans le mésenchyme et va inhiber FGF-10. La balance Shh-FGF-10 va permettre la ramification et la dichotomie bronchique. D’après Affolter et al. [61]. 